\title{
In vitro Culture and Diet of Entomopathogenic Nematodes
}

\author{
Iram Khan Tahir ${ }^{*}$, Azra Shaheen \\ Department of Zoology, Sir Syed Faculty of Sciences, Mohammad Ali Jauhar University, Rampur, Uttar Pradesh, India
}

Received January 27, 2020; Revised March 26, 2020; Accepted April 19, 2020

Copyright $\odot 2020$ by authors, all rights reserved. Authors agree that this article remains permanently open access under the terms of the Creative Commons Attribution License 4.0 International License

\begin{abstract}
The goal of this paper is to demonstrate in vitro techniques of culturing Entomopathogenic nematode and the diet on which EPN survive and flourish. The in vitro production of nematodes is essential for the commercial use of EPN. The competence of a range of media to promote growth and development of the nematodes has been evaluated. The diet consists of a base diet plus a nutritive supplement. Nematode yield is a crucial factor for successful large-scale viable production of entomopathogenic nematode. Few attempts at in vitro culture of EPNs have been undertaken. The various methods used to commence cultures are described. The diet named Kabuli gram medium constitute Nutrient broth $(0.88 \mathrm{~g})$, yeast extract $(0.32 \mathrm{~g})$, Kabuli gram $(16 \mathrm{~g})$, soybean oil $(10.40 \mathrm{ml})$, and distilled water $(60 \mathrm{ml})$ proved effective, very nutritious and appropriate for nematode culture, with this diet EPN flourish in good quantity. In vitro culturing requires more attention, nominal temperature. A small negligence will destroy the colonies of EPN in no time. However, quality and quantity of in vitro produced Entomopathogenic nematode vary considerably, depending on diet and temperature. The EPN cultured, were used for the biocontrol of Helicoverpa armigera in further study on the crops of Pisum sativum and Cicer arietenum. The production and culture of EPN had help in the biocontrol. EPN used in present and further study were Steinernema masoodi and Oscheius nadarajni.
\end{abstract}

Keywords In vitro Culture, EPN, Diet, Mass Production, Biocontrol, Cicer arietenum

\section{Introduction}

The nematode species which are endoparasitic on insect pests are broadly termed as Entomopathogenic nematodes (EPN). These nematodes are vermiform and cylindrical in shape, microscopic ranging between 0.3 and $1.0 \mathrm{~mm}$ having separate sexes and follow symbiotic relationship with bacteria, e.g. Xenorhabdus or Photorhabdus. They spend life in soil as well as in insects. After entering into insect body through natural openings, they release bacteria, which produce toxins and kill the insect by providing a good medium for multiplication of EPN. So in one way we can say that these nematodes serve as a vector for the bacteria to kill the pest thus rendering to their control.

EPN of genus Steinernema, Heterorhabditis and Oscheius are symbiotically associated with bacteria of genus Xenorhabdus and Photorhabdus respectively. These are lethal obligatory parasites; yet pose no threat to plants.

The production of insect hosts and nematodes should be optimized for maximum fitness, quality and cost efficiency. Subsequently, EPN that were fed diets with varying nutritive components were tested for virulence and reproduction capacity. Furthermore, in a general sense, our results indicate diets can be optimized for improvement in vitro EPN production efficiency. This is the first report of an EPN diet. Additionally, our studies indicate that diet may impact broader aspects of Entomopathogenic nematodes ecology and pest control efficacy. Entomopathogenic nematodes are biological control agents that are used to control a variety of economically important insect pests (Shapiro-Ilan et al., 2002b; Grewal et al., 2005). Entomopathogenic nematodes are cultured in vivo or in vitro for large-scale commercial production as well as for laboratory experimentation or field testing (Shapiro-Ilan and Gaugler, 2002; Ehlers and Shapiro-Ilan, 2005). In vitro production is accomplished in solid or liquid media. One of the components that contributes to successful in vitro production is the quality of artificial media (Shapiro-Ilan and Gaugler, 2002; Ehlers and Shapiro-Ilan, 2005).

\section{Materials and Methods}

To extract nematodes, first we took soil samples from 
different localities. We kept them separately in perforated plastics boxes. All the boxes were labeled with locality and date. Insect larvae of same size and age were picked from insect culture. We use Helicoverpa armigera (Hubner) larvae for this purpose, Corcyra cephalonica or Galleria mellonela larvae could also be used. As shown in figure 1,2, and 3 different insects were cultured for this purpose.

Picked $H$. armigera larva then released into plastic boxes. Each box may carry 4-5 larvae. After 3-4 days, dead larvae were taken out, if they are infected by EPN, they might have changed their colour to red or green. Nematodes enter the larva through their natural openings, and reside in their alimentary canal. These larvae then taken out and arranged on a watch glass, covered with watt man's filter paper. Then covered with another petri plate. Filter must be moistened. After sometime cadavers were filled with nematodes, they multiply and their population floats in the water filled in petri plate. These are juveniles of different stages and were collected in a beaker, to make formulations to be sprayed.

\section{Rearing Procedure}

\subsection{Environmental Conditions}

EPN'S natural habitat is soil. It is a difficult environment for persistence of any organism considering its complex of physical, chemical and biological components. EPN have been isolated from soils throughout the world ranging from sub-arctic to arid, temperate to tropical climatic conditions. It is assumed that in course of evolution, EPN just like any other terrestrial organisms, adopted unique survival mechanism to resist environmental extremes, unfavorable environmental conditions including absence of water, extreme temperature, lack of oxygen and osmotic stress etc.

Cold tolerance: Nematode inhabiting the soil is exposed to sub zero temperature in temperate, arctic and sub-arctic regions as well as at high altitudes. Steinernematids have been isolated at many sites from northern Europe and Canada. Heterorhabditis have been from northern Europe. EPN isolates from such cold regions indicate that they are capable of withstanding sub zero conditions. Some nematodes can even survive below freezing point at which metabolism is likely to have ceased. Cryopreservation studies demonstrated that EPN could be stored indefinitely in liquid nitrogen.

Heat tolerance: Some EPN isolated from arid region of Israel (Heterorhabditis) Egypt (S.abbasi) or tropical climate of Sri lanka were shown to be heat tolerant. Improvements of heat tolerance of EPN has been a prime goal of several workers. Recently, two new species of Steinernema, namely S.seemae and S.masoodi have been reported to be isolated from soils of pulse growing regions of Uttar Pradesh when temperature was soaring high during the summer month

Desiccation tolerance: Basically EPN are aquatic organisms and need a film of water surrounding their body in order to move. Dry condition adversely affects nematode mortality and survival. Such nematode cannot survive exposure to sunlight desiccate in field. These nematodes are capable of partial anhydrobiosis and can survive in a desiccated state for a certain period only.

Partial anhydrobiosis is usually reached following a slow rate of water loss. Some nematodes from tight coils when exposed to desiccation, resulting in slowing the rate of water loss and by reducing the area of cuticle exposed to air.

\subsection{Culturing of Nematodes (EPN) from the Soil}

Nematodes are first inoculated in to the soil where they grow and replicate we can say they reproduce autonomously to increase their generation. These nematodes have motility downwards. So when they are inoculated in to the soil they tend to move downwards into the soil. These nematodes have a life cycle of around fifteen days during which they pass from various stages including larva to adults. The infective stage is known as the juvenile stage (IJ). This stage of the EPN is Entomopathogenic nematodes can be cultured in the soil. In this process of culturing; they supposed to cause the infection into the host.

After the nematodes are inoculated into the soil they are left for some days, around 20-25 days so that they can undergo reproduction and increase in number. After that the soil samples are collected in a container.

A special apparatus is set to separate or rather filter out the nematodes from the soil. This consists of a metallic sieve, a piece of tissue paper and a collecting chamber.

1. At first the soil sample is transferred into the metallic sieve, which is covered with a tissue paper and dead insect were put on white trap (White 1927) to observe multiplication of EPN on insect body.

2. The soil is kept moist to avoid dehydration. The collecting chamber is filled with water up to a level just sufficient to touch the sieve.

3. This makes the contact between the soil sample and the water in the collecting chamber.

4. The nematodes, which always has a tendency to move downwards, passes into the water through the tissue paper.

5. The water is collected after 24 hours and the number of nematodes is count with the help of counter.

6. This extraction of nematodes continues for a few days from the same soil sample until most of the nematodes are extracted from it.

\subsection{Diet of Entomopathogenic Nematode}

Various media were tested with little modification 
depending upon locally available ingredients with varying degree of success. For in vitro production of EPN, various media have been reported.

Their ingredients are as follows:

1. Wout's medium: Nutrient $\operatorname{broth}(0.88 \mathrm{~g})$, yeast exract $(0.32 \mathrm{~g})$, soybean flour $(14.40 \mathrm{~g})$, groundnut oil $(10.40 \mathrm{~g})$, and distilled water $(60 \mathrm{ml})$.

2. Modified wout's medium: Nutrient broth $(0.50 \mathrm{~g})$, yeast extract $(0.20 \mathrm{~g})$, soybean flour $(16.00 \mathrm{~g})$, groundout oil $(12.00 \mathrm{~g})$, and distilled water $(30 \mathrm{ml})$.

3. Wheat flour medium: wheat flour $(15 \mathrm{~g})$, kabuli gram flour $(5 \mathrm{~g})$, beef extract $(5 \mathrm{~g})$, yeast extract $(6 \mathrm{~g})$, agar $1 \%(1 \mathrm{~g})$, coconut oil $(6 \mathrm{~g})$, and ditilled water $(60 \mathrm{ml})$.

4. Modified wheat flour medium: wheat flour $(15 \mathrm{~g})$, kabuli gram flour $(5 \mathrm{~g})$, beef extract $(5 \mathrm{~g})$, yeast extract $(1 \mathrm{~g})$, agar $1 \%(1 \mathrm{~g})$, groundnut oil $(10 \mathrm{~g})$, and distilled water $(60 \mathrm{ml})$.

5. Egg yolk media I: SDEY (7g), yeast extract $(2 \mathrm{~g})$, $\mathrm{NaCl}(0.8 \mathrm{~g})$, oil $(15 \mathrm{~g})$, and distilled water $(60 \mathrm{ml})$.

6. Egg yolk media II: SDEY (10g), yeast extract (5g), $\mathrm{NaCl}(0.8 \mathrm{~g})$, oil $(12 \mathrm{~g})$, and distilled water $(60 \mathrm{ml})$.

7. Egg yolk media Modified: SDEY $(7 \mathrm{~g})$, soybean flour (20g), yeast extract (2g), $\mathrm{NaCl}(0.8 \mathrm{~g})$, oil( $15 \mathrm{~g})$, and distilled water $(60 \mathrm{ml})$.

8. Dog biscuit medium: Dog biscuit (15g), yeast extract $(1 \mathrm{~g})$, peptone $(3 \mathrm{~g})$, agar $(2 \mathrm{~g})$, oil $(10 \mathrm{~g})$, and distilled water $(60 \mathrm{ml})$.

9. Modified Dog biscuit medium: Dog biscuit (20g), yeast extract $(1 \mathrm{~g})$, peptone $(0.5 \mathrm{~g})$, beef extract $(5 \mathrm{~g})$, oil $(7 \mathrm{~g})$, and distilled water $(100 \mathrm{ml})$.

In our studies, various media were tested with little modification depending upon the availability of locally ingredients with varying degree of success.

\section{Results}

The following media have been proposed, prepared and tested for the first time for this work only we named it Kabuli gram medium: Nutrient broth $(0.88 \mathrm{~g})$, yeast extract $(0.32 \mathrm{~g})$, Kabuli gram $(16 \mathrm{~g})$, soybean oil $(10.40 \mathrm{ml})$, and distilled water $(60 \mathrm{ml})$.

The ingredients were mixed and coated onto polyether polyurethane foam cut into small cubic pieces till the latter soaked in the media (1.5g of foam chips, $8-9 \mathrm{~g}$ medium, $\mathrm{w} / \mathrm{w})$. The flasks were filled with foam media impregnated water and autoclaved for $20 \mathrm{~min}$ at $121^{\circ} \mathrm{C}$ and allowed to cool. Fresh IJs extracted from the insects were used each time to avoid the prior inoculation of bacteria in the flasks. The nematodes were inoculated asceptically into the flasks (a) $1000 \mathrm{IJs} /$ Flask. The sealed flasks were incubated at $28^{\circ} \mathrm{C}$ for 25 days. The colonies of the EPN started appearing on the walls of the flasks after two weeks post inoculation. After 25 days of inoculation IJs were harvested.

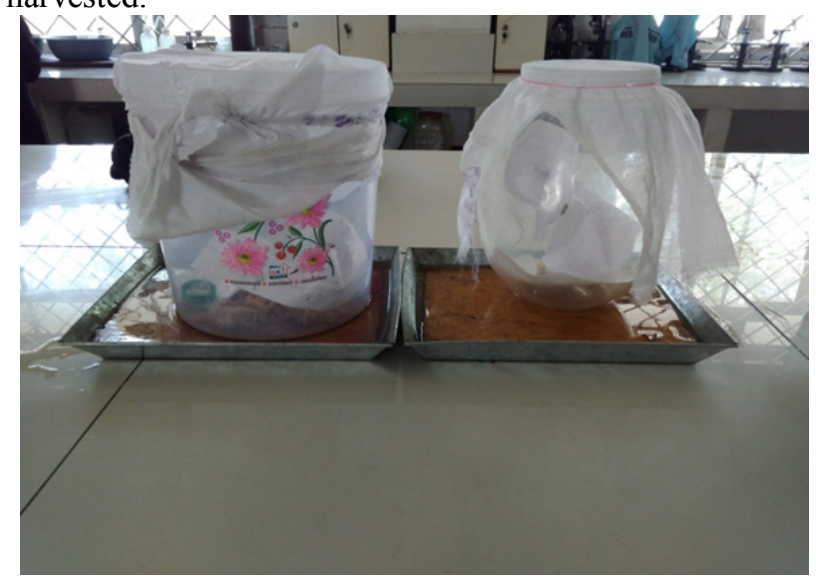

Figure 1. Rearing of Galleria mellonela in laboratory

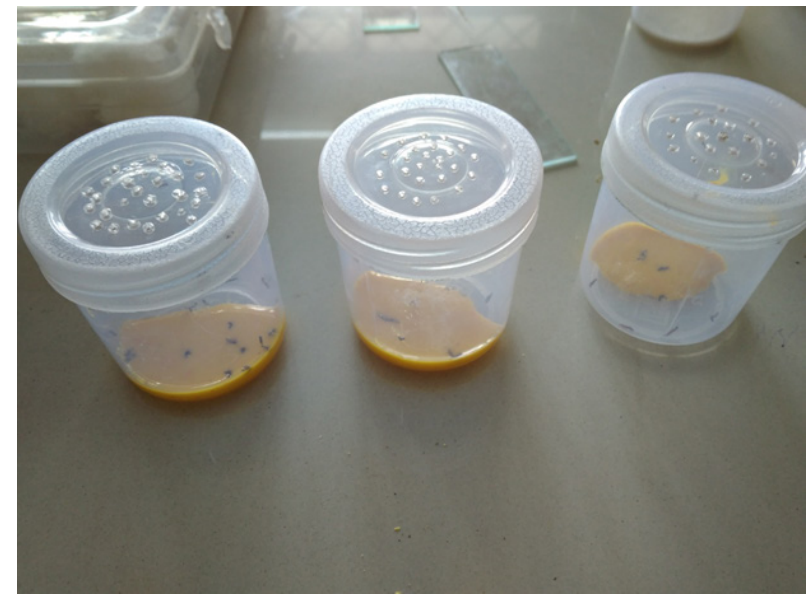

Figure 2. Rearing of Helicoverpa armigera (larvae) in laboratory

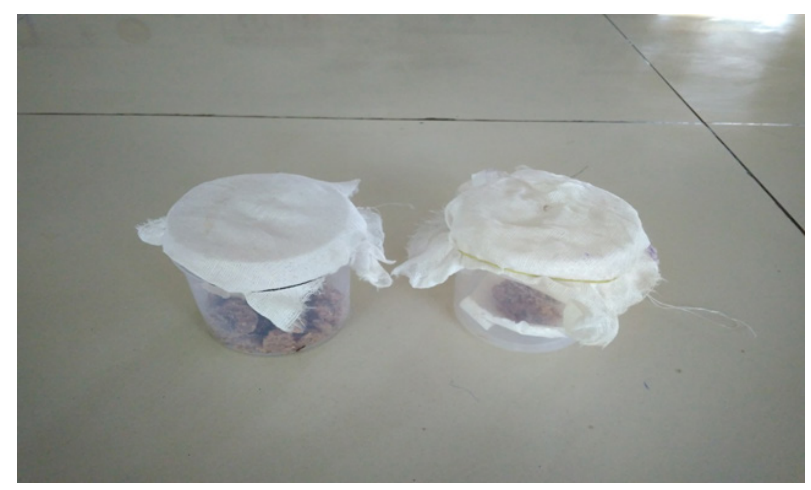

Figure 3. Rearing of Corcyra cephalonica

The foam chips were piled up a 100-mesh sieve. The sieve was placed in a pan of distilled water overnight. The nematodes sedimented were passed through a 250-mesh sieve in a pan of water. About $95 \%$ IJs migrated into $0.1 \%$ Hyamine solution, washed thrice with sterile distilled water and were collected and stored at room temperature. After concentrating nematode suspension with the help of microfiltration assembly, EPN were kept till further use. 


\section{Discussions}

The above diet Kabuli gram which was prepared for the first time in lab, proved very nutritious and it is appropriate for nematode culture. With the help of this diet EPN grow in a great quantity which made the further work easier. Ingredients used are easily available in market at affordable price and are eco-friendly. Nematodes can be extracted or separated, just by dipping foam pieces in water. Moreover, EPN produced in this diet are cold tolerant, heat tolerant and can live for long time, simply in water medium. Thus, we can transport them to faraway places, where they can be used as spray in field, by making simple formulations. Best part of all these processes is that pesticide prepared does not harm any plant or animal in any case. Additionally, host diet effects may be essential for maintaining beneficial traits during recurrent culture for laboratory or commercial purposes (Shapiro-Ilan, D. I. et al. 2012); although beneficial trait deterioration in entomopathogenic nematodes has been reported to be genetically based (Bai et al., 2005; Adhikari et al., 2009; Chaston et al., 2011), nutritional factors may still contribute to cumulative defects and trait loss (Hopper et al., 1993). The EPN Steinernema and Oscheius were used in different formulation to control the Helicoverpa armigera biologically from the fields of Pisum sativum and Cicer arietenum. Hence the work was very much effective.

\section{Acknowledgements}

Authors are grateful to their Chancellor Mohammad Azam Khan, Vice Chancellor Sultan Mohammad khan, Dean Dr. Gulrez Nizami for providing the facilities used in this study. NBAIR, Bangluru is also gratefully acknowledged for providing insect culture. Dr. Syeda Uzma Usman \& Dr. Farha Rehman for their constant and untiring efforts throughout this work.

\section{REFERENCES}

[1] Adhikari BN, Chin-Yo L, Xiaodong B, Ciche TA, Grewal PS, Dillman AR, Chaston JM, Shapiro-Ilan DI, Bilgrami AL, Gaugler R, Sternberg PW, Adams BJ. Transcriptional profiling of trait deterioration in the insect pathogenic nematode Heterorhabditis bacteriophora. BMC Genomics. 2009; 10: 609 .

[2] Bai C, Shapiro-Ilan DI, Gaugler R, Hopper KR. Stabilization of beneficial traits in Heterorhabditis bacteriophora through creation of inbred lines. Biological Control. 2005; 32: 220227.

[3] Chaston JM, Dillman AR, Shapiro-Ilan DI, Bilgrami AL, Gaugler R, Hopper KR, Adams BJ. Outcrossing and crossbreeding recovers deteriorated traits in laboratory cultured Steinernema carpocapsae nematodes. International Journal of Parasitology. 2011; 41: 801-809

[4] Ehlers, R.-U.,and Shapiro-Ilan, D. I. 2005. Massproduction. Pp. 65- 78 in P. S. Grewal, R.-U.Ehlers, and D. I. Shapiro-Ilan, eds. Nematodes as Biocontrol Agents. Wallingford, UK: CABI Publishing.

[5] Grewal, et al., 2005. Nematodes as Biocontrol Agents. Wallingford, UK: CABI Publishing.

[6] Hopper KR, Roush RT, Powell W. Management of genetics of biological-control introductions. Annual Review of Entomology. 1993; 38: 27-51.

[7] Shapiro-Ilan, D. I., and Gaugler, R. 2002. Production technology for entomopathogenic nematodes and their bacterial symbionts. Journal of Industrial Microbiology and Biotechnology 28:137-146.

[8] Shapiro-Ilan, D. I. et al., 2002b. Factors affecting commercial success: Case studies in cotton, turf and citrus. Pp.333-356 in R. Gaugler, ed. Entomopathogenic Nematology. New York: CABI.

[9] Shapiro-Ilan, D. I. et al., 2012. Optimization of a Host Diet for in vivo Production of Entomopathogenic Nematodes. Pp 264-273. The journal of Nematology. Nematol.

[10] White, G. F. 1927. A method for obtaining infective nematode larvae from cultures. Science, 66: 302- 303 . 